\title{
BMJ Open Nurse-led patient-centred intervention to increase written advance directives for outpatients in early-stage palliative care: study protocol for a randomised controlled trial with an embedded explanatory qualitative study
}

\author{
Katia Iglesias (D) , ${ }^{1}$ Catherine Busnel, ${ }^{2}$ Florian Dufour, ${ }^{3}$ Sophie Pautex, ${ }^{4}$ \\ Laurence Séchaud ${ }^{5}$
}

To cite: Iglesias K, Busnel C, Dufour F, et al. Nurse-led patient-centred intervention to increase written advance directives for outpatients in early-stage palliative care: study protocol for a randomised controlled trial with an embedded explanatory qualitative study. BMJ Open 2020;10:e037144. doi:10.1136/ bmjopen-2020-037144

- Prepublication history for this paper is available online. To view these files, please visit the journal online (http://dx.doi. org/10.1136/bmjopen-2020037144).

Received 22 January 2020 Revised 03 August 2020 Accepted 14 August 2020
Check for updates

(C) Author(s) (or their employer(s)) 2020. Re-use permitted under CC BY-NC. No commercial re-use. See rights and permissions. Published by BMJ.

For numbered affiliations see end of article.

Correspondence to Dr Katia Iglesias;

katia.iglesias@hefr.ch

\section{ABSTRACT}

Introduction Discussing the evolution of lifethreatening diseases and end-of-life issues remains difficult for patients, relatives and professionals. Helping people discuss and formalise their preferences in end-of-life care, as planned in the Go Wish intervention, could reduce health-related anxiety in the advance care planning (ACP) and advance directive (AD) process. The aims of this study are (1) to test the effectiveness of the Go Wish intervention among outpatients in early-stage palliative care and (2) to understand the role of defence mechanisms in end-of-life discussions among nurses, patients and relatives.

Methods and analysis A mixed-methods study will be performed. A cluster randomised controlled trials with three parallel arms will be conducted with 45 patients with chronic progressive diseases impacting life expectancy in each group: (1) Group A, Go Wish intervention for patients and their relatives; (2) Group A, Go Wish intervention for patients alone and (3) Group B, for patients (with a waiting list), who will receive the standardised information on ADs (usual care). Randomisation will be at the nurse level as each patient is referred to one of the 20 participating nurses (convenience sample of 20 nurses). A qualitative study will be conducted to understand the cognitive and emotional processes and experiences of nurses, patients and relatives confronted with end-of-life discussions. The outcome measurements include the completion of ADs (yes/no), anxiety, quality of communication about endof-life care, empowerment, quality of life and attitudes towards ADs.

Ethics and dissemination The study protocol has been approved by the Human Research Ethics Committee of the Canton of Geneva, Switzerland (no. 2019-00922). The findings will be disseminated to practice (nurses, patients and relatives), to national and international scientific conferences, and peer-reviewed journals covering nursing science, psychology and medicine.

Trial registration number NCT04065685.
Strengths and limitations of this study

- Favourable setting to investigate death-related thoughts: proximal and continuous long-term relation between patients and nurses, nurses trained about advance directives, institutional support, extra time for end-of-life discussions.

- New knowledge about cognitive and emotional processes experienced when discussing end-of-life issues, while simultaneously taking into account the different stakeholders (patients/relatives/ nurses) through qualitative and quantitative data (triangulation).

- New knowledge about the efficacy of nurse-led complex advance care planning (ACP) intervention based on the Go Wish tool.

- Information about efficacy only investigated at midterm for patients and relatives (with the last measure being 3 months after the inclusion).

- General practitioners as stakeholders of the ACP process are not actively involved.

\section{BACKGROUND}

In Switzerland, only $10 \%$ of the deaths per year occur suddenly and unexpectedly, and about two-thirds of those deaths are from an illness after receiving medium to longterm medical care for their life-limiting conditions. ${ }^{1}$ Much of the population has an invested interest in the process of advance care planning (ACP) for end-of-life care and in the use of advance directives (ADs) ${ }^{2} \mathrm{ADs}$ are legal documents in which patients record decisions about medical treatments they want (or do not want) to receive. Since 2013, with the Swiss Adult Protection Law enacted, healthcare professionals are legally obligated to enquire whether a patient has ADs in place. 
ADs allow people to indicate their therapeutic choices and care options to healthcare professionals and to choose a surrogate, in case of loss of capacity and discernment. The public considers ADs to be useful ${ }^{3}$ and a tool for improving care. ${ }^{45}$ ADs are aimed at preventing conflicts in the decision processes among patients, close relatives and healthcare teams, especially in critical situations. ${ }^{5-7}$

However, despite ADs' perceived utility and the political and institutional campaigns that promote them, ${ }^{8}$ interventions to increase the prevalence of legally completed ADs have produced few significant increases in ADs' uptake and use in Switzerland, ${ }^{89}$ as in most countries where they exist. ${ }^{10-12}$ For instance, about 5\%-14\% of Swiss adults have completed ADs. ${ }^{18-101314}$ Concerns about the low use of ADs have focused on several explanations, such as a lack of knowledge, misconceptions and their complexity. ${ }^{12} 1516$ Recent research shows that even when people are informed about ADs, a majority of them do not formalise their choices by writing ADs. ${ }^{11}{ }^{14}$ This raises questions about how best to encourage patients to engage in ADs' elaboration and how health professionals could support such patients.

\section{From ADs to ACP}

Current developments in anticipated decision making have shifted from the traditional completion of ADs towards ACP. ACP is based on broad aims to clarify personal goals, values, religious and/or cultural beliefs, and options of care through repeated encounters, which can be considered as a complex intervention. ${ }^{17-21}$ It is seen as a continuous development of communication among patients, close relatives and health professionals, with the aim to define a common orientation for care and treatment. ${ }^{22}$ ADs are now seen as a possible part of the ACP process. Some recent systematic reviews ${ }^{22-25}$ have highlighted the heterogeneity of ACP interventions, settings, stakeholders, designs, measures and outcomes. Houben $e t a l^{23}$ showed the efficacy of ACP interventions for different populations through randomised controlled trials (RCT), specifically for the completion of ADs and the occurrence of end-of-life discussions. However, it is unclear which elements of ACP are effective or how to best promote its implementation in practice. Using similar designs but including older people, Weathers $e t$ $a l^{25}$ concluded that ACP has positive effects with respect to symptom management, quality of care and the number of ADs. Nevertheless, well-conducted RCTs that evaluate ACP interventions are lacking, regardless of the setting. Brinkman-Stoppelenburg et $a l^{22}$ also found evidence of how ACP affects the quality of end-of-life care and underlined the benefits of complex ACP interventions in comparison to written documents alone. Furthermore, for the quality of end-of-life communication, Walczak et $a l^{24}$ noticed that the evidence was variable, depending on the quality of the designs. Finally, Kermel-Schiffman and Werner ${ }^{11}$ showed in a recent systematic review that nurses (and also lay people) lacked or had inappropriate knowledge of ACP in general, even if they had sufficient knowledge about ADs. According to these authors, this knowledge gap needs to be improved by developing intervention programmes about the topic and overcoming barriers that might limit engagement in ACP with endof-life issues among professionals, patients and close relatives.

\section{The ACP intervention}

Therefore, based on the Medical Research Council framework of developing and evaluating complex interventions, ${ }^{26}$ we first conceptualised and developed the ACP intervention for patients and relatives by relying on an integrative review of literature describing various theoretical models, possible interventions and favourable conditions. ${ }^{27}$ Based on this review, we found a new research avenue using card games as a non-threatening way to start a conversation about end-of-life. The Go Wish ${ }^{27-30}$ tool was chosen because it is a patient-centred approach to help people discuss their preferences and options for end-of-life care, and then formalise them in ADs. The tool promotes collaborative partnership and shared decision making with health professionals. It is a 'serious' game of 36 cards, each presenting a statement that refers to personal needs, values and beliefs about end-of-life care. The statements are considered as important because they correspond with descriptors of 'a good death'. 3132 The statements are related to four main dimensions: (1) oneself, (2) care (technical, physical, relational and spiritual), (3) family and close relatives and (4) the context and organisation of end-of-life.

In addition, based on this review, we found that the intervention should take place in long-term care instead of in hospital care, due to the shortened stays and they are exposed to a multitude of professionals. Therefore, we identified residents in nursing homes as the best target.

Second, a pilot study with a pre/postsingle-group design was conducted on 14 nurses and 39 dyads (residents/relatives) in five nursing homes to test feasibility and acceptability of a nurse-led complex ACP intervention based on the Go Wish tool. ${ }^{33}$ It was accepted with good rates of satisfaction, comfort and agreement with the procedures by all stakeholders. The study confirmed the initial content and format of the ACP intervention. It permitted fine-tuning of the data collection procedures. We concluded the ACP intervention could be more beneficial to people in early-stage palliative care, such as those with chronic degenerative conditions that are reducing their life expectancy, those receiving care outside the hospital setting and in their natural environment or those who have less cognitive impairments than people in nursing homes.

The Go Wish intervention is characterised by low literacy barriers and by a process where the patient initiates and guides discussions on themes they want to develop. It means that a patient's own readiness is respected and reflected in their engagement in the process, such as defined in the Prochaska's transtheoretical model. ${ }^{34}$ This model proposes five steps relevant to change and is a 
frequently used theory in ACP. It offers a framework that specifies the different strategies to favour a process of intentional change behaviour. At each stage, the tailored interventions allow the passage from a non-interest to an intention and finally the realisation of an action; so, in our case the development of an ACP until the elaboration of ADs. ${ }^{155-37}$ Despite its success, however, Prochaska's transtheoretical model has been harshly criticised. ${ }^{38}$ In particular, this model strongly relies on conscious human decision making and rationality while neglecting the weight of the unconscious processes. ${ }^{39}$ However, these unconscious mechanisms (ie, defence mechanisms) could explain reluctance to formalise ADs and emerge when patients, their close relatives and health professionals experience end-of-life thoughts when considering $\mathrm{ACP}$ and ADs.

\section{The terror management health model}

The terror management theory $\left(\mathrm{TMT}^{40-42}\right)$ is a strong candidate for gaining such insights. The TMT is based on the assumption that humans are confronted with an awareness that they will inevitably die. This awareness, when mixed with the striving for survival, can produce a paralysing anxiety that requires management. According to Becker, ${ }^{43}$ self-esteem buffers the anxiety caused by an awareness of the inevitability of death. Greenberg $e t a t^{42}$ extended this initial model and developed the TMT to better understand psychological mechanisms, helping people to manage their potentially paralysing anxiety. The TMT proposes a dual-defence model in which individuals cope with the fear of their own death through proximal and distal defence mechanisms that help to preserve their well-being. On one hand, proximal defence is engaged when people are placed in a context that makes deathrelated thoughts conscious (eg, when facing a direct threat). In such a context, individuals tend to remove these cognitions from their focal awareness (eg, postpone decisions related to health). On the other hand, distal defence is activated when death-related thoughts are not, or no longer, in their focal attention but at the fringe of consciousness (eg, after suppressing deathrelated thoughts from one's consciousness). In such a context, an anxiety-buffer system prevents death-related thoughts from gaining consciousness. This anxiety-buffer system predicts that people will seek to give sense to their life and the world through (A) self-esteem enhancement, (B) the maintenance of close personal relationships and (C) an expression or defence of their cultural worldview. Goldenberg and Ardnt ${ }^{44}$ later introduced the terror management health model (TMHM) to specifically study and understand health decisions through the TMT's lens and mechanisms. ${ }^{45} 46$ TMHM predicts that death-related thoughts and subsequent existential anxiety trigger defence mechanisms, which can result in healthy intentions or healthy behaviours. ${ }^{45} 47$ Anxiety can be generated in situations such as the ACP process and while writing $\mathrm{ADs}^{44}$ because 'being diagnosed with a serious illness (or) receiving or providing medical treatment ${ }^{48}$ for example, reminds people of their own mortality. ${ }^{15}$ For instance, to best prevent threat-avoidance outcomes the result from postponing ADs' completion, one can apply TMHM principles and help people reduce their death-related thoughts by promoting components of the anxiety-buffer system. Since these components are integrated into the Go Wish tool, which focuses on individual's important life dimensions, personal relationships with close relatives/ health professionals and self-determination, the TMHM predicts that death-related thoughts should be better managed, resulting in less anxiety and less reluctance to complete ADs.

Grounded in the TMHM, we want to assess the efficacy of the Go Wish intervention as compared with the standardised information on ADs commonly provided to outpatients in early-stage palliative care.

\section{STUDY AIMS}

The first objective is to test the efficacy of the Go Wish intervention for increasing the proportion of completed ADs among outpatients receiving early-stage palliative care services as compared with usual care (ie, standardised information on ADs).

The second objective is to explore the roles of TMHM defence mechanisms in of end-of-life discussions among nurses, patients and relatives.

\section{METHODS/DESIGN \\ Study design}

To answer our research questions, we planned two separate but interrelated studies with a mixed-methods approach for the second aim of the research. The first study will test the efficacy of the Go Wish intervention as compared with the usual care: standardised information on ADs. To test the intervention's efficacy, a cluster RCT with three parallel arms will be conducted (with a waiting list for the control group). The three arms are group A, the intervention group, (1) with patients and their relative and (2) with patients without a relative; and Group B, a control group receiving the usual care, specifically the standardised information on ADs.

In the second study, we will analyse how the TMHM defence mechanisms are present in nurses, patients and relatives when experiencing end-of-life discussions. Therefore, we developed a qualitative methodology based on the grounded theory. The main goal is to understand and explain in-depth cognitive and emotional processes and experiences of the stakeholders in the first study; and which elements articulate those mechanisms.

\section{Setting}

The study will take place at the Geneva Institution for Homecare and Assistance (imad), Switzerland. The imad provides healthcare, assistance in daily living and help for relatives (if they exist) to enable people requiring assistance to stay at home. The imad's professionals include 
680 nurses divided into 44 teams. In 2016, 16947 people received the imad's services as a consequence of illness, disability or loss of independence. Three-quarters of the imad's patients are at least 65 years old $(27 \%$ aged between 65 and 79 years, and $49 \%$ aged over 80 years). Many suffer from chronic degenerative conditions that affect their life expectancy.

This setting is particularly appropriate to investigate death-related thoughts. In fact, several factors are known as barriers in the promotion of ACP and ADs: lack of knowledge about ADs, lack of time, lack of confidence, discomfort of healthcare professionals, lack of institutional support. ${ }^{49}$ This setting is optimal first because of the relation of confidence between nurses and their patients. Each nurse is referent of patients (ie, regular face-to-face visits with each patient). Second, all nurses involved in the study will be trained about ADs to demystify them, provide them the necessary knowledge to explain and give information on ADs and to help them feel more comfortable with end-of-life issues. Furthermore, this study is supported by the imad and all the nurses will be given the necessary time to promote ACP and ADs.

\section{Population and sample}

\section{Population}

To increase the proportion of patients with completed ADs, it is essential to account for the anxiety of not only the patient who writes it, but also those who have to talk about it. Therefore, three populations are of interest in this study: imad nurses, imad patients with chronic disease impacting their life expectancy (early-stage palliative care) and relatives of these patients (ie, close relatives, friends or informal caregivers). For a question of language, 'nurses' will refer to imad nurses and 'patients' to imad patients.

We plan to recruit 20 nurses (a convenience sample). Through these nurses, we plan to recruit 135 patients and around 65 relatives over the 15 months for the first study (for sample sizes, see the 'Sample size' section). Within these samples, for the second study, we plan to select randomly between 5 and 10 participants (depending on the data saturation) in each group (around 15-30 patients, 10-20 relatives and 10-20 nurses).

\section{Inclusion criteria}

- Nurses: permanent employees with a diploma in nursing, fluent in French and able to provide informed consent.

- Patients: at least 18 years old with chronic degenerative conditions that could become fatal, such as advanced cancer, chronic obstructive pulmonary disease (COPD), stage IV cardiac heart failure or multimorbidity, with severe impacts on daily life; able to speak, read and understand French; followed by nurses at least once a week; capacity for discernment and the ability to provide informed consent.

- Relatives: designated by the patient; at least 18 years old; able to speak, read and understand French; capacity for discernment; and able to provide informed consent.

\section{Exclusion criteria}

- Nurses: temporary employees or refusal to provide informed consent.

- Patients: existing ADs, terminal care, existing diagnosis of cognitive disorder or sensory motor impairment documented in the patient file related to memory loss or disturbances of speech that would not allow for a constructive exchange or refusal to provide informed consent.

- Relatives: an imad professional or refusal to provide informed consent.

\section{Recruitment procedure \\ Nurses}

Nurses will be recruited through an official announcement of the study within the imad. First, the imad's investigator will present the research to the operational directors, then each operational director will inform the nursing managers in charge of services who will communicate to their nurses. Nurses will inform their nursing managers of their interest in participating and their name will be communicated to the research team. The first 20 eligible nurses to answer the announcement will be enrolled in the study. In case of nurses' drop-outs or no sufficient patients with the inclusion criteria, a new announcement to involve nurses will be made.

\section{Patients}

Each nurse involved in the study will screen their patients and identify those who are eligible. Once the eligible patients are defined, the nurses will inform their patients of their involvement in the ongoing study. They will give their patients an informational flyer and explain that the study will give them extra time to discuss their healthcare preferences in both groups (without explaining further the study). The nurses will ask eligible patients if they will allow a research team's member (a coinvestigator or a study nurse) to come next time with them to present the study in further detail. The nurses will note the patients' responses (yes/no) and submit the information to the research team. If the patients agree to this contact, then a research team's member will accompany the nurse during the next visit. A research team's member will explain the study to the patients and provide them with the information sheet and the consent form to read. To allow enough time for each patient to consent, a new appointment will be made with a research team's member, during which the patients will sign the consent form and answer the first questionnaire with a research team's member. All eligible patients of the 20 nurses will be invited to participate in the study until 135 patients are enrolled. For the second study, a random sample of the RCT's patients will be invited (between five to ten in each arm until data saturation). If patients agree to participate in the second study, then a research team's member will explain the 
study to the patients, give them the information sheet and the consent form to read and make an appointment for the semistructured interview.

\section{Relatives}

All patients involved in the study will be asked by a research team's member if they want to participate with a relative or not. If the patients mention a relative, then a research team's member will take note of the relative's name, their relation (relative, friend, neighbour, etc), and the phone number. A research team's member will contact the relative by phone (while mentioning the patient's agreement), explain the study and send the relative an information sheet and a consent form to read. If the relative is interested, then the research team member will make the next appointment, when the relative will sign the consent form. The questionnaires will be answered with a research team's member either face to face or by phone, depending the relative's wishes. For the second study, a random sample of the RCT's relatives will be invited (between five to ten in each arm until data saturation). If the relatives agree to participate to the qualitative study, a research team's member will explain the study to the relatives, give them an information sheet and the consent form to read, and make an appointment for the semistructured interview.

\section{Procedure for the nurses}

Nurses will follow a common standardised training on ADs ('Joint training') provided by an experienced clinical specialist nurse (shorter version of the usual imad's workshop (1.5 hours instead of 3) delivered since 2017).

After the joint training, the nurses will be randomised into two groups (group A or group B). A stratified randomisation will be run to account for the socioeconomic levels of the imad teams' locations. If two or more nurses of a same imad team want to participate in the study, then to prevent contamination within the team, they will be randomised into the same group (clustered randomisation).

After the randomisation, group A will receive a 3-hour training session on the Go Wish tool. ${ }^{28}$ This training will be delivered to group $\mathrm{B}$ at the end of the study. This specific training covers four points: (1) the theoretical and legislative aspects of ADs (refreshment of training provided within imad) and distinction from ACP, (2) presentation of Go Wish and intervention steps and characteristics of each encounter, (3) simulation of the three encounters between participants with nurses taking turns in each role, and (4) collective debriefing and provision of memos for each step reminding nurses how to introduce the intervention, give instructions, document patients' choices and conclude the encounter. In addition, a written summary of the attitudes to be promoted and elements to be taken into account in carrying out the intervention will be given to the nurses afterwards on the basis of the comments and proposals made during the training. The training for group B will be 1-hour long and related to the delivery of the standardised information on ADs to patients. For further details, see the Study Intervention section.

Once trainings are given, each nurse will meet one of the investigators to ensure that he or she has acquired the new skills and to remind him or her about the research steps and the formal aspects needed in term of data quality for the research project. The nurse will be required to: (A) collect and transmit information related to their group to the research team; (B) document and justify the possible adjustments to the intervention according to the context and/or the characteristics of the persons being cared for and their relatives; $(\mathrm{C})$ foster collaboration within the care and research team and (D) understand and respect the study protocol by following the schedule for the phases of the process and the instructions for carrying out the interviews. Throughout the study, the trainer will ensure continuous monitoring of the intervention's delivery to identify and overcome any difficulties and to ensure the intervention is standardised.

Both groups of nurses will answer online questionnaires (taking around $20 \mathrm{~min}$ ) at baseline and 1,3 and 12 months after inclusion (for details, see 'Outcomes' section and table 1). One month after the end of patients' inclusion, 10 to 20 nurses will be invited for a semistructured qualitative interview of 45-60 min. For the full procedure for the nurses, see figure 1 .

\section{Procedure for patients}

The allocation of the patients to group A or B will depend on their nurse's allocation. The nurses will propose the

Table 1 Questionnaires and qualitative interviews: time since patients' inclusion for nurses

\section{Nurses}

\begin{tabular}{|c|c|c|c|c|c|}
\hline & \multicolumn{5}{|l|}{ Nurses } \\
\hline & Baseline & 1 month & 3 months & 12 months & $\begin{array}{l}1 \text { month after end of patients' } \\
\text { inclusion }\end{array}$ \\
\hline Attitudes towards ADs & $x$ & $x$ & $x$ & $x$ & \\
\hline Anxiety & $x$ & $x$ & $x$ & $x$ & \\
\hline Qualitative interviews & & & & & $\mathrm{X}$ \\
\hline
\end{tabular}

ADs, advance directives. 


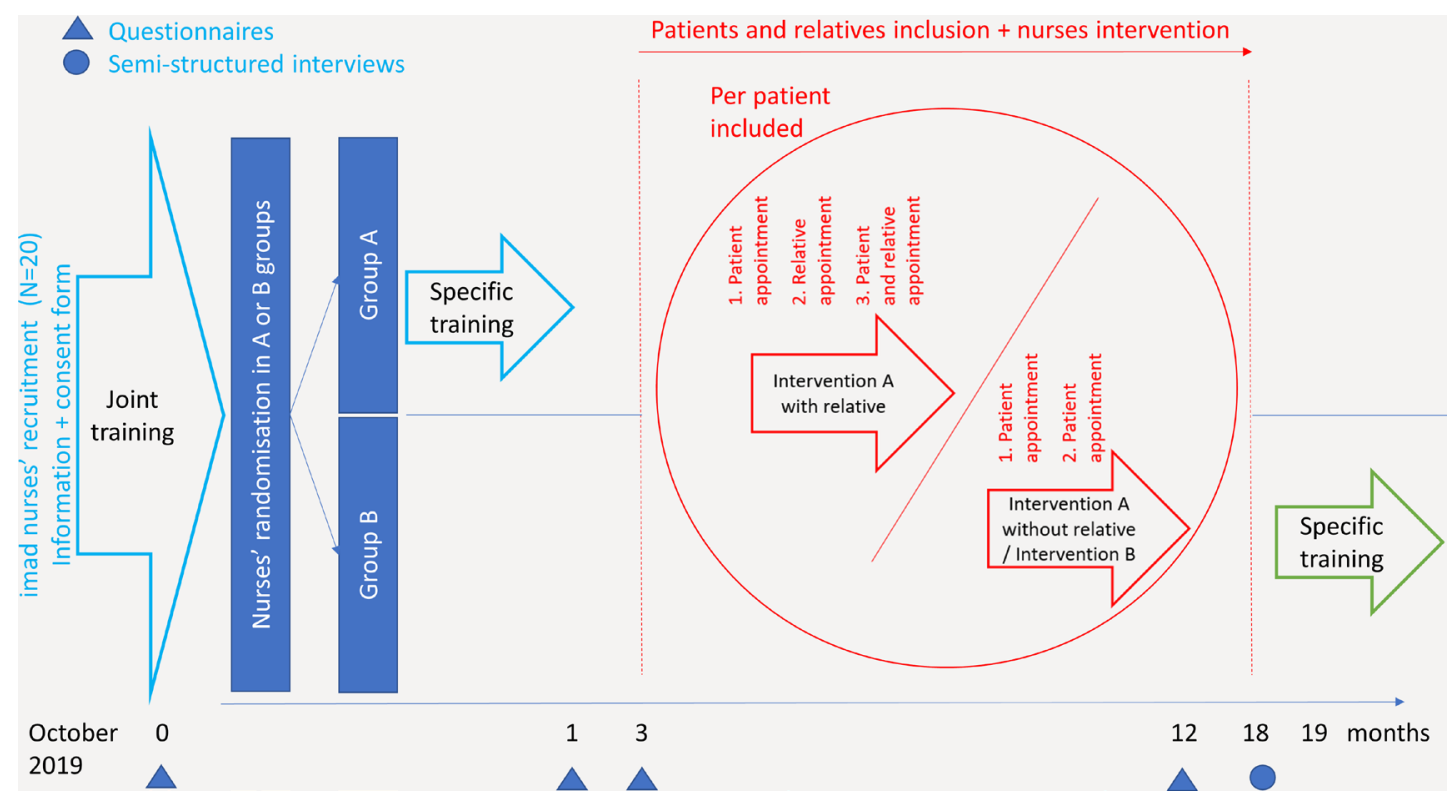

Figure 1 Procedure for nurses of groups A and B. (A): Go Wish tool; (B): usual care: standardised information on advance directives. Colours: (1) blue; nurses as participants of the study, (2) red; nurse's appointments with patients and relatives depending on the group and (3) green; after the study.

study to their eligible patients during a care visit. If a patient is interested, a research team's member will come and explain the study to the patient at the end of care during the following nurse visit. If patients agree once they are informed, they will sign a consent form and be asked to complete questionnaires twice (see the 'Outcomes' section and table 2) - at baseline and 3 months after inclusion-with the help of a research team's member. Questionnaires are estimated to take $45-60 \mathrm{~min}$ to complete at each time point.

After the baseline questionnaires, the nurse will arrange appointments with the participant to give the intervention. The patients of Group A will use the Go Wish tool throughout two 1-hour appointments (the second appointment being jointly held with the relative, if the participant has designated one). The patients of group B will have two appointments lasting $15-20 \mathrm{~min}$ to discuss the standardised information on ADs.
Six months after inclusion in the study, the nurses will ask the patients if they want to elaborate on their ADs and propose that a member of the research team comes to their house. During the appointment, patients will be presented with the common existing documents on ADs, comprising sections such as motivations and personal values, therapeutic representative and options of care. They will choose their preference, fill in the document, and define the way they want to share it and with whom (imad file, close relative, general practitioner (GP), etc). One month afterward, a subsample of patients will be invited to a $45 \mathrm{~min}$ semistructured qualitative interview conducted by a research team's member.

The patients in group B will receive the Go Wish intervention between one and ten months after the semistructured interview, depending on the moment of inclusion in the study. For the full procedure for the patients, see figure 2 .

Table 2 Questionnaires and qualitative interviews: time since inclusion for patients and relatives

\begin{tabular}{|c|c|c|c|c|c|c|c|c|}
\hline & \multicolumn{4}{|l|}{ Patients } & \multicolumn{4}{|l|}{ Relatives } \\
\hline & Baseline & 3 months & 6 months & 7 months & Baseline & 3 months & 6 months & 7 months \\
\hline Sociodemographic data & $x$ & & & & $\mathrm{X}$ & & & \\
\hline $\begin{array}{l}\text { Quality of communication about } \\
\text { end-of-life care }\end{array}$ & $x$ & $x$ & & & & & & \\
\hline Written ADs & & & $x$ & & & & & \\
\hline Qualitative interviews & & & & $x$ & & & & $x$ \\
\hline
\end{tabular}

ADs, advance directives. 


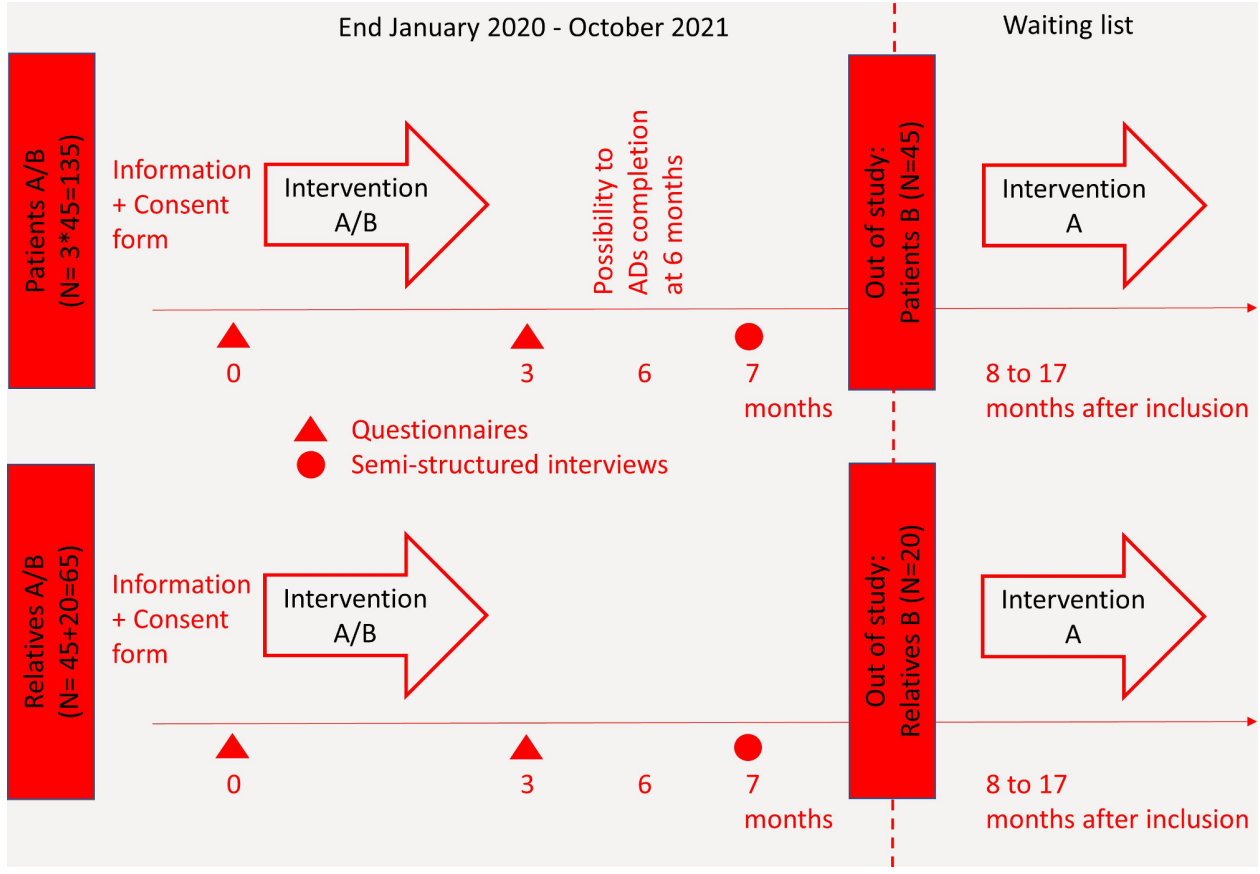

Figure 2 Procedure for the patients and relatives of groups A and B. (A): Go Wish tool; (B): usual care: standardised information on advance directives.

\section{Procedure for the relatives}

The allocation of the relatives to group A or B will depend on the patients' nurse allocation. The patient included in the study can invite a relative. Once a relative is named, a member of the research team will contact the relative to propose the study. If relatives, once informed, signs the consent form, they will be asked to complete several questionnaires (see the 'Outcomes' section and table 2) at baseline and 3 months after inclusion, with the help of a research team's member, if needed. Questionnaires are estimated to take $25-35$ min to complete at each time point.

For the relatives in group A, after the baseline questionnaire, the nurse will set two appointments with them lasting 1 hour each using the Go Wish tool (the second appointment being held jointly with the patients). The relatives of group B will have no contact with the nurse for the study. Seven months after inclusion into the study, 10-20 relatives will be invited to a semistructured qualitative interview lasting $30-45 \mathrm{~min}$ by a research team's member. Relatives in group B will receive the Go Wish intervention between 1 and 10 months after the ADs' possible completion, depending on the moment of inclusion in the study. For the full procedure for relatives, see figure 2 .

\section{Study intervention}

\section{Group A}

The nurse-led intervention will be tested with the serious game Go Wish. In the intervention, the patients will meet their nurses twice. During the first session, nurses will ask patients to (A) read each card carefully; (B) sort the cards into three piles corresponding to high, medium or low importance for them, and (C) rank the cards in the high-importance pile from most to least important. If the patients sort fewer than ten cards into the highimportance pile, they will move cards from the second pile (comprising the statements of medium importance) to reach a final number of ten cards. Even though cards propose sentences, their interpretation is broad (eg, 'To be treated the way I want' or 'To maintain my dignity'). The broadness of cards and the neutrality of the nurses' intervention will allow for free expression; nurses will not interfere in the choice of cards and prioritisation or give their interpretation of the cards, even if the patients request it. Nurses will have the responsibility to lead the procedure and adopt a listening, reformulating posture to facilitate the choices' expression of patients. They will give the cards to patients to be discovered at their own rhythm, without pressure, orientation or comment. For relatives, a similar procedure will be used, but centered on the patients' preferences. During the last interview, nurses will propose that patients comment on their priorities by explaining them and considering the consequences in terms of therapeutic options. They will reiterate the previously expressed care and treatment preferences and link them to patients' health status and symptoms as well as current treatments (with their advantages and disadvantages) and possible evolution. For relatives, nurses will play the role of facilitator of exchanges for greater mutual understanding within the dyad. The objective will be to discuss the independently selected priorities and identify and explore the similarities and differences. The intervention will end with documentation of the patients' values, wishes or preferences to put into the patients' files and potential subsequent appointments with a member of the research team to name surrogates or elaborate on ADs. 
Each appointment will take an average of 1 hour. However, nurses will take the necessary time for each participant, and the whole intervention will be completed between 3 and 6 weeks.

\section{Group B}

After providing their care, the nurses will provide a leaflet containing the standardised information on ADs, which will permanently be in the patient's health record booklet. They will explain the information contained in the leaflet and give enough time for the patients to ask any questions. One week afterward, the nurses will once again hand the leaflet to the patients and make themselves available for any questions.

At any moment between the intervention and 6 months later, for both groups, if a patient wishes to write down information about his or her care, the nurse will ask the patient if he or she wants to elaborate ADs and propose that a member of the research team should come to his/ her house. The patients will be encouraged to inform their GP if they write any ADs.

\section{Hypotheses and research question}

\section{Hypothesis 1a}

The percentage of patients with ADs will be higher in the group of patients who will receive the Go Wish intervention jointly with a relative than in the group of patients who will receive the standardised information on ADs.

\section{Hypothesis 1b}

The percentage of patients with ADs will be higher in the group of patients who will receive the Go Wish intervention without integrating a relative than in the group of patients who will have the standardised information on ADs.

\section{Hypothesis 2}

Standardised information on ADs, instead of the Go Wish intervention, will increase anxiety among nurses, patients and relatives.

\section{Hypothesis 3}

Nurses' attitudes towards ADs will differ by the type of training (Go Wish vs standardised information on ADs).

\section{Hypothesis 4}

The quality of communication between nurses and patients about end-of-life care will be higher for patients in the Go Wish group compared with the group receiving standardised information on ADs.

\section{Hypothesis 5}

Empowerment will be greater among patients in the Go Wish group compared with those receiving the standardised information on ADs.

\section{Hypothesis 6}

Quality of life will be greater for patients and relatives in the Go Wish group compared with those receiving the standardised information on ADs.

\section{Research question}

What were cognitive and emotional processes and experiences of the nurses, patients and relatives during the study and how did they operate together when confronted with end-of-life discussions?

\section{Outcomes}

Primary outcome point

ADs

The completion of ADs (yes/no) will be measured by the number of ADs completed by patients 5 months after the intervention (around 6 months after their inclusion).

\section{Secondary outcomes}

Secondary outcomes will be measured at the patient, relative and nurse levels (see tables 1 and 2).

\section{Anxiety}

Anxiety will be assessed with the validated French version of the Positive and Negative Affect Scale (PANAS) ${ }^{50}$ It consists of two 10-item scales to measure mood: PANAS (with two subscales: afraid and upset). The items are rated on a 10-point Likert scale.

\section{Attitudes towards $A D s$}

Attitudes towards ADs will be assessed with the validated Knowledge Attitudes and Practice Behaviours Questionnaire (KAP), ${ }^{51}$ translated and then back-translated into French. ${ }^{52}$ The KAP is 52 -item scale comprising five dimensions: (A) demographic and practical characteristics (11 items); (B) knowledge about ADs (12 items); (C) attitudes when faced with ADs (18 items), split up into beliefs (eight items), subjective norms (six items) and perceived control (four items); (D) practices during the discussions (four items) and (E) other information about practices (seven items). The questions related to knowledge about ADs cares (12 items) were excluded because they were not relevant to the research questions. The items are rated on a 5-point Likert scale.

\section{Quality of end-of-life communication}

The communication between the patients and nurses will be assessed with the validated Quality of Patient-Clinician Communication about End-of-Life Care measure ${ }^{5354}$ This 19-item scale comprises two subscales: (A) global communication competencies of the clinician (seven items) and (B) specific competencies determining the quality of the communication about end-of-life care (12 items). The items are rated on a 10-point Likert scale. Translation and back translation will be run.

\section{Patient empowerment}

Patient empowerment will be assessed with the validated French version of the Healthcare Empowerment Questionnaire. ${ }^{55}$ This 10 -item scale evaluates the degree of empowerment perceived by the patient from the care received and health services on a 4-point Likert scale. 


\section{Quality of life}

- The patients' quality of life will be assessed with the validated French version of the McGill Quality of Life Revised. ${ }^{56}$ This 14-item scale is composed of four subscales (plus one general quality-of-life question): physical (three items), existential (four items), psychological (four items) and social (three items). The items are measured on an 11-point Likert scale.

- For the relatives, quality of life will be assessed with the validated French version of WHO Quality of Life Brief. ${ }^{57}$ This 26 -item scale is based on satisfaction questions across four domains of quality of life-physical health (seven items), psychological health (six items), social relationships (three items) and environment (eight items)-plus two general questions. Items are rated on a 5-point Likert scale in reference to the last 2 weeks. A percentage rating within each domain will be computed with scores ranging from 0 (lowest quality of life) to 100 (highest quality of life), as defined by the instrument use.

\section{Other variables}

For patients: age, gender, level of education, main disease (s), imad admission criteria and admission date.

For relatives: age, gender, level of education and relationship with the patient (partner, child, friend, neighbour, etc).

For nurses: age, gender, level of education, time as a nurse, time as a nurse at imad and activity rate (items from the first scale of the KAP).

\section{Statistical analysis \\ Sample size}

The percentage of the population with completed ADs in Switzerland has been estimated at around $2 \%{ }^{89}$ We anticipate that 5 months after inclusion in the study, around $5 \%$ of the patients of Group B will have ADs and around $25 \%$ of the patients of group A will have ADs. With a significance level of 0.05 , a power of 0.8 , and an attrition rate of $20 \%$, we need to include 45 patients in each group to show the superiority of intervention A on the proportion of patients with completed ADs, as compared with intervention B.

Go Wish was first developed for patients only ${ }^{28}$ and later for dyads (patients and relatives), ${ }^{27}$ to increase its beneficial effects. For ethical reasons, and to not stigmatise patients without relatives, the recruitment for the intervention group will be doubled to 45 patients with relatives and 45 patients without relatives. We will test the intervention's efficacy for those with (hypothesis 1a) and without relatives (hypothesis 1b). Group B will include 45 patients (with or without relatives, with around 20 relatives). We will recruit a total of 135 patients receiving imad services and around 65 relatives. Twenty nurses are needed to recruit 135 patients (with an average of 25 patients referred to each nurse during the 15 months of the study; $60 \%$ in early-stage palliative care and a $50 \%$ response rate)'.
For the second study, we will randomly select 5-10 patients, relatives and nurses in each group until data saturation.

\section{Quantitative data analyses}

First, descriptive statistics (frequencies and percentages for categorical data, means, SD, medians and IQR intervals for quantitative data) will be run at each time point for each group and for the whole sample. In addition, non-responses and patient dropouts will be analysed. Second, bivariate associations will be computed to compare the groups at baseline (Pearson $\chi^{2}$ test, Fisher's exact tests, t-tests and Mann-Whitney tests, depending on the variables' distributions). Third, to test the intervention's impact on the number of written ADs (hypothesis 1), a logistic regression will be run controlling for sociodemographic characteristics and health-related variables. Fourth, to assess how psychological factors (our secondary outcomes) among the nurses, patients and relatives impact the primary outcome, we will run a stepwise logistic regression on ADs for the explanatory variables. Fifth, to test the impacts of intervention/ training on nurses' attitudes towards ADs; anxiety among nurses, patients and relatives; patients' communication and empowerment; and patients' and relatives' quality of life (hypotheses 2-6), linear mixed-effects models with participants (patients, relatives and/or nurses) as a random effect will be run to analyse the changes over time for each outcome. The models will be tested on the available information, as no listwise deletion is needed for such analyses. The effects of time, groups and their interactions will be tested while controlling for sociodemographic characteristics and health-related variables. Sixth, to control for a possible cluster effect of patients nested within nurses, as a sensitive analysis, a generalised linear mixed model with nurses as a random effect for hypothesis 1 and a linear mixed-effects model with nurses as an additional random effect for hypotheses 2-6 will be run and compared with previous analyses. Statistical analyses will be carried out using R and STATA (V.12, StataCorp), with a significance level of $p=0.05$, under the supervision of a statistician.

The statistical analyses will be performed on the available data, and no missing data will be imputed. As our survey will be administered face to face with a research team's member, and based on prior experience, we expect the number of missing values to be low.

Handling missing data: Concerning the patients, we will compare non-respondents to respondents using the available information: sex, age, duration of follow-up at imad and illness.

The sample size was increased by $20 \%$ to compensate for expected drop-outs. Furthermore, drop-outs will be analysed to identify potential biases in the sampling.

\section{Qualitative data analyses}

The qualitative methodology used in the second study is based on the constructivist grounded theory approach. ${ }^{559}$ 
This theoretical framework proposed an inductive and circular design of research to retrieve the qualitative information that will explain in-depth processes operating on the phenomena studied. Following the work of Charmaz ${ }^{58}$ the qualitative approach will be implemented for a theory-building goal: to develop a substantive theory explaining the cognitive and emotional processes underlying the beliefs, attitudes and experiences of the nurses, patients and relatives experiencing end-of-life discussions.

The following methodology will be used: First, for our fieldwork will be conducting in-depth interviews with the participants of the three target groups. All the interviews will be audio-recorded, anonymised and verbatim transcribed. The principle of saturation will determine the number of interviews to conduct. Second, will be the analysis of the data. Interview transcriptions will be analysed with the support of software Atlas.ti.

The coding will be made up in three $\operatorname{stages}^{58}$ : (1) initial coding (applying a line-by-line coding to identify phenomena/themes using action language as codes that are helping to interpret the text in a new way); (2) focused coding (initial codes compared and grouped into categories, until core categories by data immersion and memoswriting are identified) and (3) axial coding (specification of categories' properties and dimension, identification of links and reassembling data).

The analytical procedure is based on constant comparisons: data collection, doing memos (to keep track of concepts and their properties) and diagrams (to visualise the relation between concepts), comparing similarities and differences for identifying, developing and integrating concepts until a theory is generated. At this step, we will perform a literature review to contextualise the identified categories and key concepts within the existing research literature. These steps will be run in a cyclical way until the information saturation.

Rigour of data collection and analysis will be attempted by following Charmaz's principles ${ }^{58}$ : credibility (eg, peer debriefing, memos-writing, audit trail of analytical decisions), originality (eg, review of memos), resonance (eg, present the categories to participants for validation) and usefulness (sharing results to people outside the study into the practice). All of the analyses will be conducted by two independent researchers, and the results will be discussed to reach agreement.

The findings will provide a theoretical context for the quantitative findings and will help us to better interpret our findings (triangulation analyses are planned).

\section{Ethics and dissemination}

\section{Consent}

All procedures in studies involving human participants will be performed in accordance with the ethical standards of the institutional and/or national research committee and with the 1964 Declaration of Helsinki and its later amendments, or comparable ethical standards. The protocol, information letters, questionnaires and informed consent forms of the study have been approved by the Human Research Ethics Committee of the Canton of Geneva, Switzerland (no. 2019-00922). No adverse events or side effects are expected for the participants. Informed consent will be obtained from all participants included in the study.

Each participant will be informed that participation in the study is voluntary and that they may withdraw from the study at any time. Participants who withdraw their consent or express the desire to end the research interview will be withdrawn from the study. Data collected up to that point will be analysed in order not to compromise the study's validity. We will ensure that participants who prematurely withdraw from the study do not need assistance. Premature withdrawal from the study will be documented. Due to the low level of risk for the participants, no early termination of the study for safety reasons is anticipated. If the study budget allows it, participants who withdraw from the study will be replaced, to ensure sufficient power.

\section{Ethical considerations}

The population is a potentially vulnerable population with specific psychosocial needs to consider regarding their serious illness. Therefore, we have taken the following steps to ensure that patients' needs will be fully met and respected:

1. The intervention for group A will be proposed to group B at the end of the study 1 .

2. All of the materials (the consent form, procedures and questionnaires) were previously tested by patients corresponding to the targeted population.

3. A research team's member will provide help with completing the questionnaires.

4. If needed, the appointments can be spread out to respect the patients' fatigability.

5 . The extra costs of the study will be entirely supported by the study grant. No extra costs will be charged to the patients or to health insurance.

The relatives will also form part of the study, so we have ensured that

1. The intervention of group A will be proposed to Group $\mathrm{B}$ at the end of the measures.

2. All of the materials (the consent form, procedure and questionnaires) were previously tested by relatives corresponding to the targeted population.

3. A research team's member will provide help with completing the questionnaires.

4. The relatives will not be charged.

For the nurses,

1. The specific training of group A will be proposed to Group B at the end of the measures.

2. All of the materials (the information form, procedure and questionnaires) were previously tested by nurses corresponding to the targeted population.

3. The time required to participate in the various steps of this research (the training, intervention, questionnaires, semistructured interviews and extra time with patients and relatives) will be guaranteed by imad 
during the professionals' work hours, as imad will receive specific funding from the study grant so that nurses will participate in this research.

\section{Risks}

The risk of serious adverse consequences as a result of study participation is low. However, participants may experience emotional discomfort related to end-of-life discussions and may have concerns about the confidentiality of the sensitive information collected during the interviews. The patients can be reassured that only researchers with the appropriate skills will collect data and interact with the patients and relatives, to reduce any discomfort and provide a secure environment for the discussions.

If patients/relatives experience significant distress, they will be referred to a competent professional (psychologist; GP) by the nurses or a research team's member, which will be part of the training.

Although the data to be collected during the study are personal and longitudinal, the data cannot be anonymous. However, the participants will be reassured that their responses will be anonymised, completely confidential and not shared with any other third party without their permission. At the end of the intervention, the nurses will ask what information discussed during the meetings can be share and where/with whom (imad file, GP, etc).

The project leader will be responsible for monitoring the data, ensuring protocol compliance and conducting safety reviews. The researchers have commissioned a trial steering group composed of experts: a physician and a specialised nurse in palliative care, a psychologist and a statistician. This group will also be responsible for data monitoring and ethical review to ensure any decisions made will be in the best interests of the patients, relatives, and nurses. During the review process, the investigators and the steering committee will evaluate whether the study should continue unchanged, require modification/amendment, continue or be closed to enrolment. In the unlikely event that adverse events or unanticipated problems occur, a member of the research team will immediately inform the project leader and the principle investigator. The project leader will report any serious events to the research ethical committee within 7 days. She will also submit a report evaluating the relationship between the reported event and the methods of health-related personal data collection within the project as well as proposals on how to proceed with the project.

\section{Data confidentiality}

No data in the planned research will be obtained through laboratory tests or invasive procedures. The trial and participant data will be handled with the utmost discretion and will only be accessible to authorised personnel who require the data to fulfil their duties within the scope of the study. On the case report forms and other studyspecific documents, participants will be only identified by a unique participant number.
The generation, transmission, storage and analysis of health-related personal data within this project will strictly follow the current Swiss legal requirements for data protection and will be performed according to Ordinance ClinO Art. 18.

All master lists of names (or other identifiable information), personal identification numbers and consent forms will be stored in a locked office. Access to master lists and signed consent forms will be restricted to the members of the research team.

All electronic data will be recorded using only personal identification numbers and will be stored separately from any identifiable information in password-protected computers on secured servers.

Quantitative data will be collected using Research Electronic Data Capture (REDCap).

Qualitative interview data will be recorded using a digital recorder. Audio files and the interviews transcribed into Word files will be labelled with only the patient's identification (ID) number and will be transferred to passwordprotected computers for electronic storage on secured servers in the institution of the first author (HEdS-FR). Once the transcriptions have been verified, the audio files will be destroyed.

\section{Dissemination plan}

Findings will be disseminated to the clinic (nurses, patients and relatives) and to national and international scientific conferences and peer-reviewed journals in nursing science, psychology and medicine.

\section{Patient and public involvement}

The sounding group of patients, relatives and nurses with similar characteristics as those of the stakeholders of our research, revised all the proposed documents of the study for patients, relatives and nurses.

\section{DISCUSSION}

The current study is designed to provide evidence concerning the efficacy of a nurse-led intervention on ADs completion and also regarding experiences with endof-life discussions among nurses, patients and relatives. This study project will offer both clinical and theoretical contributions. From a clinical point of view, by testing a nurse-led patient-centred intervention on realisation of ADs, we can ensure that the care provided to outpatients meets their expectations and needs by circumventing implementation-defence mechanisms related to anxiety caused by the ADs' context, as suggested by the TMHM. We also expect to improve professionals' communication skills, particularly outside hospital environments, and to support professional practice in initiating discussions about end-of-life care. By focusing on goals, values and beliefs, rather than on specific treatments or interventions, the proposed intervention may help to alleviate the resistance arising from death-related thoughts. It should decrease patient anxiety and increase empowerment, 
quality of communication and the proportion of patients with ADs.

From a theoretical point of view, this study will enrich the ongoing reflection on the low use of ADs under conditions in which known barriers are diminished by placing it in the TMHM framework. This will help us understand the cognitive and emotional processes as well as experiences among nurses, patients and relatives when confronted with end-of-life discussions and to explain the roles of the TMHM defence mechanisms for stakeholders, thereby allowing us to improve this aspect.

\section{Limitations}

This study has some limitations related to time restrictions and patients' vulnerability. First, information about efficacy will be only investigated at mid-term for patients and relatives (with the last measure being 3 months after inclusion). Second, we will not observe the quality of end-of-life communication directly; it will be only assessed through self-reported measures for patients and semistructured interviews with stakeholders. Therefore, fidelity of the intervention could not be assessed. Third, GPs, as stakeholders of the ACP process, will not be actively involved.

\section{Implication}

For the participating patients, we expect the benefits to include (A) clarification of their care options according to their wishes, preferences and values; (B) promotion of the development of ACP and an increased number of formalised ADs; (C) strengthened participation in decision-making processes concerning them (empowerment) and (D) increased well-being. The benefits to both patients and their relatives include (A) strengthened communication and partnerships and (B) promotion of the surrogate function. For professionals, the benefits include (A) training on use of the Go Wish tool and (B) strengthened end-of-life communication and capacity to develop ACP and ADs. For all of the participants, the benefit will be the promotion and anticipation of a shared decision process in end-of-life situations.

Since the risks of adverse effects (ie, psychological discomfort) associated with participation in the study are low, we believe that the benefits of the study will outweigh its risks.

\section{Ethics approval and consent to participate}

All procedures in studies involving human participants will be performed in accordance with the ethical standards of the institutional and/or national research committee and with the 1964 Declaration of Helsinki and its later amendments, or comparable ethical standards. The study's protocol, information letters, questionnaires and informed consent forms have been approved by the Human Research Ethics Committee of the Canton of Vaud, Switzerland (no. 2019-00922). No adverse events or side effects are expected for the participants. Informed consent will be obtained from all participants included in the study.

\section{Author affiliations}

${ }^{1}$ School of Health Sciences (HEdS-FR), HES-SO University of Applied Sciences and Arts Western Switzerland, Friourg, Switzerland

${ }^{2}$ Geneva Institution for Homecare and Assistance (imad), Geneva, Switzerland ${ }^{3}$ School of Management and Engineering Vaud (HEIG-VD), HES-SO University of Applied Sciences and Arts Western Switzerland, Yverdon, Switzerland

${ }^{4}$ Division of Palliative Medicine, Department of rehabilitation and geriatrics, University Hospitals Geneva, Geneva, Switzerland

${ }^{5}$ Geneva School of Health Sciences, HES-SO University of Applied Sciences and Arts Western Switzerland, Geneva, Switzerland

Acknowledgements The authors wish to thank Paul N'Dri Konan for his initial participation in the study's development; Dawn Carnes and Gora Da Silva Rocha for their sustain and helpful feedback; and the patients, relatives and nurses in our sounding group for their feedback on the material for the study participants.

Contributors $\mathrm{Kl}$ and LS wrote the manuscript. All of the authors critically reviewed the manuscript for important intellectual content. The study design and research proposal were mainly developed by $\mathrm{KI}$ and LS. CB, SP and FD made substantial contributions to the study's conception and design. The intervention was developed by $\mathrm{KI}$ and LS. All of the authors agree to be accountable for all aspects of the work, in ensuring that questions related to the accuracy or integrity of any part of the work are appropriately investigated and resolved. All of the authors have read and approved the final manuscript.

Funding This study is funded by the Swiss Academy of Medical Sciences, grant no. PC 12/18.

\section{Competing interests None declared.}

Patient and public involvement Patients and/or the public were involved in the design, or conduct, or reporting, or dissemination plans of this research. Refer to the Methods section for further details.

Patient consent for publication Not required.

Provenance and peer review Not commissioned; externally peer reviewed.

Open access This is an open access article distributed in accordance with the Creative Commons Attribution Non Commercial (CC BY-NC 4.0) license, which permits others to distribute, remix, adapt, build upon this work non-commercially, and license their derivative works on different terms, provided the original work is properly cited, appropriate credit is given, any changes made indicated, and the use is non-commercial. See: http://creativecommons.org/licenses/by-nc/4.0/.

ORCID iD

Katia Iglesias http://orcid.org/0000-0003-1308-1631

\section{REFERENCES}

1 Binder J, von Wartburg L. Stratégie nationale en matière de soins palliatifs 2010-2012. Office fédéral de la santé publique OFSP, Conférence suisse des directrices et directeurs cantonaux de la santé (CDS), eds 2009.

2 Haesen S, Shaw D. Directing citizens to create advance directives. Swiss Med Wkly 2018;148:w14628.

3 Clements JM. Patient perceptions on the use of advance directives and life prolonging technology. Am J Hosp Palliat Care 2009;26:270-6

4 Bond WF, Kim M, Franciskovich CM, et al. Advance care planning in an accountable care organization is associated with increased advanced directive documentation and decreased costs. J Palliat Med 2018;21:489-502.

5 Silveira MJ, Kim SYH, Langa KM. Advance directives and outcomes of surrogate decision making before death. N Engl J Med 2010;362:1211-8.

6 Detering KM, Hancock AD, Reade MC, et al. The impact of advance care planning on end of life care in elderly patients: randomised controlled trial. BMJ 2010;340:c1345.

7 Tierney WM, Dexter PR, Gramelspacher GP, et al. The effect of discussions about advance directives on patients' satisfaction with primary care. J Gen Intern Med 2001;16:32-40.

8 Brzak N, Papadaniel Y, Berthod M-A. Les proches au cœur des décisions médicales de fin de vie : anticiper, représenter, hésiter. Anthropologie et Santé 2016. 
9 Pautex S, Gamondi C, Philippin Y, et al. Advance directives and endof-life decisions in Switzerland: role of patients, relatives and health professionals. BMJ Support Palliat Care 2018;8:475-84.

10 Pautex S, Herrmann FR, Zulian GB. Role of advance directives in palliative care units: a prospective study. Palliat Med 2008;22:835-41.

11 Kermel-Schiffman I, Werner P. Knowledge regarding advance care planning: a systematic review. Arch Gerontol Geriatr 2017;73:133-42.

12 Seymour J, Gott M, Bellamy G, et al. Planning for the end of life: the views of older people about advance care statements. Soc Sci Med 2004;59:57-68

13 Office fédéral de la santé publique OFSP. La planification anticipée concernant la santé, en particulier en cas d'incapacité de discernement (« Advance Care Planning »). Cadre général pour la Suisse [Internet], 2018. Available: https://www.palliative.ch/fileadmin/ user_upload/palliative/bilder_neu/180212_Rahmenkonzept Gesundheitl_Vorausplanung-1.pdf

14 Cattagni Kleiner A, Santos-Eggimann B, Seematter-Bagnoud L. Directives anticipées, représentant thérapeutique et mandat pour cause d'inaptitude: connaissance, utilisation et perception chez les personnes âgées [Internet]. 80. Lausanne: Institut universitaire de médecine sociale et préventive (IUMSP), 2016. http://www.iumsp.ch/ Publications/pdf/rds263_fr.pdf

15 Fried TR, Bullock $\mathrm{K}$, lannone $\mathrm{L}$, et al. Understanding advance care planning as a process of health behavior change. J Am Geriatr Soc 2009;57:1547-55.

16 Kavalieratos D, Corbelli J, Zhang D, et al. Association between palliative care and patient and caregiver outcomes: a systematic review and meta-analysis. JAMA 2016;316:2104-14.

17 MacKenzie MA, Smith-Howell E, Bomba PA, et al. Respecting choices and related models of advance care planning: a systematic review of published evidence. Am J Hosp Palliat Care 2018;35:897-907.

18 Selwood A, Senthuran S, Blakely B, et al. Improving outcomes from high-risk surgery: a multimethod evaluation of a patient-centred advanced care planning intervention. BMJ Open 2017;7:e014906.

19 Kirchhoff KT, Hammes BJ, Kehl KA, et al. Effect of a disease-specific advance care planning intervention on end-of-life care. J Am Geriatr Soc 2012;60:946-50.

20 Song M-K, Donovan HS, Piraino BM, et al. Effects of an intervention to improve communication about end-of-life care among African Americans with chronic kidney disease. Appl Nurs Res 2010;23:65-72.

21 Waldrop DP, Meeker MA. Communication and advanced care planning in palliative and end-of-life care. Nurs Outlook 2012;60:365-9.

22 Brinkman-Stoppelenburg A, Rietjens JAC, van der Heide A. The effects of advance care planning on end-of-life care: a systematic review. Palliat Med 2014;28:1000-25.

23 Houben CHM, Spruit MA, Groenen MTJ, et al. Efficacy of advance care planning: a systematic review and meta-analysis. J Am Med Dir Assoc 2014;15:477-89.

24 Walczak A, Butow PN, Bu S, et al. A systematic review of evidence for end-of-life communication interventions: who do they target, how are they structured and do they work? Patient Educ Couns 2016;99:3-16.

25 Weathers E, O'Caoimh R, Cornally N, et al. Advance care planning: a systematic review of randomised controlled trials conducted with older adults. Maturitas 2016;91:101-9.

26 Craig P, Dieppe P, Macintyre S, et al. Developing and evaluating complex interventions: the new medical Research Council guidance. BMJ 2008;337:a1655

27 Séchaud L, Goulet C, Morin D, et al. Advance care planning for institutionalised older people: an integrative review of the literature. Int J Older People Nurs 2014;9:159-68.

28 Menkin ES. Go wish: a tool for end-of-life care conversations. J Palliat Med 2007;10:297-303

29 Lankarani-Fard A, Knapp H, Lorenz KA, et al. Feasibility of discussing end-of-life care goals with inpatients using a structured, conversational approach: the go wish card game. J Pain Symptom Manage 2010;39:637-43.

30 Séchaud L. Besoins prioritaires en fin de vie pour les personnes âgées et LeuRS proches. Revue internationale de soins palliatifs 2014:29:14-15

31 Steinhauser KE, Christakis NA, Clipp EC, et al. Factors considered important at the end of life by patients, family, physicians, and other care providers. JAMA 2000;284:2476-82.

32 Heyland DK, Dodek P, Rocker G, et al. What matters most in endof-life care: perceptions of seriously ill patients and their family members. CMAJ 2006;174:627-33.
33 Séchaud L. La planification anticipée du projet thérapeutique (PAPT) auprès des personnes âgées vivant en établissement médico-social. [Internet] [PhD in nursing sciences]. University of Lausanne, 2014. https://serval.unil.ch/notice/serval:BIB B5C396840129

34 Prochaska JO, DiClemente CC. Transtheoretical therapy: toward a more integrative model of change. Psychother Theory Res Pract 1982;19:276-88.

35 Fried TR, O'Leary JR. Using the experiences of bereaved caregivers to inform patient- and caregiver-centered advance care planning. J Gen Intern Med 2008;23:1602-7.

36 Schickedanz AD, Schillinger D, Landefeld CS, et al. A clinical framework for improving the advance care planning process: start with patients' self-identified barriers. J Am Geriatr Soc 2009;57:31-9.

37 Sudore RL, Schickedanz AD, Landefeld CS, et al. Engagement in multiple steps of the advance care planning process: a descriptive study of diverse older adults. J Am Geriatr Soc 2008;56:1006-13.

38 Armitage CJ. Is there utility in the transtheoretical model? Br J Health Psychol 2009;14:195-210.

39 West R. Time for a change: putting the Transtheoretical (stages of change) model to rest. Addiction 2005;100:1036-9.

40 Greenberg J, Pyszczynski T, Solomon S, et al. Role of consciousness and accessibility of death-related thoughts in mortality salience effects. J Pers Soc Psychol 1994;67:627-37.

41 Solomon S, Greenberg J, Pyszczynski T. A terror management theory of social behavior: the psychological functions of self-esteem and cultural worldviews. In: Zanna MP, ed. Advances in experimental social psychology. Elsevier, 1991: 93-159.

42 Greenberg J, Pyszczynski T, Solomon S. The causes and consequences of a need for self-esteem: a terror management theory. In: Baumeister RF, ed. Public self and private self. New York, NY: Springer New York, 1986: 189-212.

43 Becker E. The denial of death. New-York: Academic Press, 1973.

44 Goldenberg JL, Arndt J. The implications of death for health: a terror management health model for behavioral health promotion. Psychol Rev 2008;115:1032-53.

45 Arndt J, Cook A, Goldenberg JL, et al. Cancer and the threat of death: the cognitive dynamics of death-thought suppression and its impact on behavioral health intentions. J Pers Soc Psychol 2007;92:12-29.

46 Taubman-Ben-Ari O, Findler L. Proximal and distal effects of mortality salience on willingness to engage in health promoting behavior along the life span. Psychol Health 2005;20:303-18.

47 Vail KE, Juhl J, Arndt J, et al. When death is good for life: considering the positive trajectories of terror management. Pers Soc Psychol Rev 2012;16:303-29.

48 Johnstone M-J. Bioethics, cultural differences and the problem of moral disagreements in end-of-life care: a terror management theory. J Med Philos 2012;37:181-200.

49 Blackwood DH, Walker D, Mythen MG, et al. Barriers to advance care planning with patients as perceived by nurses and other healthcare professionals: a systematic review. J Clin Nurs 2019;28:4276-97.

50 Gaudreau P, Sanchez X, Blondin J-P. Positive and negative affective states in a performance-related setting: testing the factorial structure of the panas across two samples of French-Canadian participants. Eur J Psychol Assessment 2006;22:240-9.

51 Zhou G, Stoltzfus JC, Houldin AD, et al. Knowledge, attitudes, and practice behaviors of oncology advanced practice nurses regarding advanced care planning for patients with cancer. Oncol Nurs Forum 2010;37:E400-10.

52 Boukar F. Des directives anticipées la planification anticipée du projet thérapeutique: connaissances, attitudes et pratiques des infirmières domicile [Master thesis]. Lausanne, La Source: Institut et Haute Ecole de la Santé, 2014.

$53 \mathrm{Au} \mathrm{DH}$, Udris EM, Engelberg RA, et al. A randomized trial to improve communication about end-of-life care among patients with COPD. Chest 2012;141:726-35

54 Engelberg R, Downey L, Curtis JR. Psychometric characteristics of a quality of communication questionnaire assessing communication about end-of-life care. J Palliat Med 2006:9:1086-98.

55 Gagnon M, Hibert R, Dubé M, et al. Development and validation of an instrument measuring individual empowerment in relation to personal health care: the health care Empowerment questionnaire (HCEQ). Am J Health Promot 2006;20:429-35.

56 Cohen SR, Sawatzky R, Russell LB, et al. Measuring the quality of life of people at the end of life: the McGill quality of life QuestionnaireRevised. Palliat Med 2017;31:120-9.

57 Skevington SM, Lotfy M, O'Connell KA, et al. The world Health organization's WHOQOL-BREF quality of life assessment: psychometric properties and results of the International field trial. A report from the WHOQOL group. Qual Life Res 2004;13:299-310. 
58 Charmaz K. Constructing grounded theory: a pratical guide through qualitative analysis. 2nd edn. London: Sage Publications, 2014.
59 Corbin J, Strauss A. Basics of qualitative research: techniques and procedures for developing Grounded theory. 5th edn. Thousand Oaks, CA: Sage Publications, 2015. 